\title{
基于成像光谱仪的冬小麦苗期冠层叶绿素密度监测
}

谭海珍 $^{1,2}$ 李少昆 $^{1,2,{ }^{*}} \underset{\text { 王克如 }}{1,2}$ 谢瑞芝
军臣

( ${ }^{1}$ 新疆兵团绿洲生态农业重点开放实验室, 新疆石河子 $832003 ;^{2}$ 中国农业科学院作物科学研究所 / 国家农作物基因资源与基因改 良重大科学工程, 北京 100081)

摘 要: 利用自主研发的 MSI200 型成像光谱仪对冬小麦苗期叶绿素密度进行遥感监测, 并与 ASD Fieldspc FR2500 型非成像地物光谱仪测定数据相比。结果表明, 两种仪器所得 R720、FD660、FD550、TCARI、GNDVI、PPR (550, 450)、 NRI 等光谱特征参量均与叶绿素密度显著相关, 拟合度较高, 说明 MSI200 型成像光谱仪测定的作物光谱数据是可靠 的。对篮选出的模型进行检验, ASD Fieldspc FR2500 模型的预测精度达 86.61\% 92.79\%, MSI200 达 91.26\% 95.54\%, 其中 PPR 对叶绿素密度预测精度最高, RMSE 分别为 0.8391 和 0.0979 。与传统非成像地物光谱仪相比, 成像光谱仪 能够提取纯冠层光谱信息, 所得模型精度高、误差小, 笁选的植被指数和特征波段对于航天、航空遥感器的定标有重 要意义。

关键词: 冬小麦; 苗期; 冠层; 叶绿素密度; MSI200 型成像光谱仪; ASD Fieldspc FR2500 型非成像地物光谱仪

\section{Monitoring Canopy Chlorophyll Density in Seedlings of Winter Wheat Using Imaging Spectrometer}

TAN Hai-Zhen ${ }^{1,2}$, LI Shao-Kun ${ }^{1,2, *}$, WANG Ke-Ru ${ }^{1,2}$, XIE Rui-Zhi' ${ }^{2}$, GAO Shi-Ju², MING Bo ${ }^{1,2}$, YU Qing ${ }^{2}$, LAI Jun-Chen ${ }^{1,2}$, LIU Guo-Qing ${ }^{1,2}$, and TANG Qiu-Xiang ${ }^{2}$

$\left({ }^{1}\right.$ Key Laboratory of Oasis Ecology Agriculture of Xinjiang Construction Crops, Shihezi 832003, Xinjiang; ${ }^{2}$ Institute of Crop Sciences / National Key Facility for Crop Gene Resources and Genetic Improvement, Chinese Academy of Agricultural Sciences, Beijing 100081, China)

\begin{abstract}
Imaging spectrometer is a new kind of remote sensing sensor, which receives images of ground objects and their spectrum components. It has great potentials in accurately quantitative analysis with remote sensing of high spatial and spectral resolutions. MSI200 is an imaging spectrometer manufactured by the authors. It was used in this study to monitor canopy chlorophyll density in winter wheat (Triticum aestivum L.) at seedling stage in comparison with ASD Fieldspc FR2500, a well-known non-imaging spectrometer. Correlation analysis showed that R720, FD660, FD550, TCARI, GNDVI, PPR (550, 450), and NRI obtained by the 2 spectrometers were both significantly correlated $(P<0.01)$ with canopy chlorophyll density. This primarily indicated the reliability of MSI200. The precisions of predicted model on chlorophyll density were 91.26-95.54\% and 86.61-92.79\% for MSI200 and ASD Fieldspc FR2500, respectively. Among these parameters, PPR was the best one to monitor canopy chlorophyll density with root mean square error (RMSE) of 0.0979 and 0.8391 for MSI200 and ASD Fieldspc FR2500, respectively. Compared with the traditional non-imaging spectrometers, MSI200 predicted better the canopy chlorophyll density in wheat with smaller errors, and the selected wavelengths were important to spaceflight and airborne remote sense.
\end{abstract}

Keywords: Winter wheat; Seedling; Canopy; Chlorophyll density; Imaging spectrometer MSI200; Non-imaging spectrometer ASD Fieldspc FR2500

高光谱成像光谱仪简称成像光谱仪(imaging spectrometer)，在对目标物成像的同时测量其波谱 特性，将目标物辐射特性与波谱特性结合为图谱特
性，或称图像立方体(3D)。其光谱分辨率高、波段连 续性强, 能得到地物真实的反射波谱信息。叶绿素 密度是进行作物农情监测、生产管理和产量估计的 
重要指标。利用光谱技术对其进行诊断, 方便、快 速、无破坏性, 在生产中具有较大应用价值。吉海 彦等 ${ }^{[1]}$ 利用高光谱技术, 在 400 750 nm的光谱范围 内建立了小麦冠层叶绿素含量与反射光谱的模型, 预测值与真实值的相关系数达 0.898 , 相对标准偏差 为 $13.6 \%$ 。赵祥等 ${ }^{[2]}$ 应用偏最小二乘回归方法，在 350 1 $060 \mathrm{~nm}$ 波段范围内构建了冬小麦冠层波谱与 叶绿素含量的回归模型, 具有较高的反演精度。这 些研究都是基于传统非成像地物光谱仪, 所获冠层 光谱数据均为混合光谱, 虽然通过构建植被指数等 方法能减小土壤等背景因素的干扰，但模型的预测 精度依然受到限制。高光谱成像仪在作物研究中的 报道较少且仅限于机载成像光谱仪。宫鹏等 ${ }^{[3]}$ 利用 机载高光谱成像仪数据实现了区别属于同一种地物 的不同类别的花旗松与美国巨杉; Curran等 ${ }^{[4]}$ 利用 AVIRIS(机载可见光成像光谱仪)数据分析出植被生 物化学参数(叶绿素、 $\mathrm{N}$ 、木质素和纤维素)的含量并 编制了调查图。有关近地高光谱成像仪的应用则少 见报道。本课题组与北京欧普特科技有限公司合作 开发了MSI200 型高光谱成像仪, 该仪器通过调制滤 光器得到不同波长的电磁波谱, 通过高性能 $\mathrm{CCD}$ 线 阵进行图像和光谱信息采集, 与传统的高光谱仪在 结构和原理上不同。为了明确成像光谱仪与传统光 谱仪在测定作物光谱中的异同及在仪器使用时应注 意的问题, 更好地定量解析航空和卫星平台的成像 光谱数据蕴涵的作物信息, 本研究以近地高光谱成 像仪对小麦冠层叶绿素监测, 为促进高光谱成像仪 在作物定量遥感中的应用, 提高作物理化参数的遥 感估测水平和今后更好地应用卫星成像光谱数据进 行作物监测提供了依据。

\section{1 材料与方法}

\section{1 试验设计}

试验于 2007 年在中国农业科学院作物科学研 究所中圑场 $\left(39^{\circ} 57^{\prime} 55^{\prime}\right.$ N $\left.N, 116^{\circ} 19^{\prime} 46^{\prime \prime} \mathrm{E}\right)$ 进行。供试 地块土壤为潮土，0 20 cm土层含有机质 $1.16 \%$, 碱 解氮 $42.6 \mathrm{mg} \mathrm{kg}^{-1}$, 有效磷 $26.5 \mathrm{mg} \mathrm{kg}$, 速效钾 $139.4 \mathrm{mg} \mathrm{kg}^{-1}$ 。选择目前生产中主推的 5 个冬小麦品 种为供试种, 其中京 411 和CA9554 为紧凑型, 中优 9507 和京 9428 为松散型, 京冬 8 号为中间过渡型。 小区面积 $8 \mathrm{~m} \times 4 \mathrm{~m}, 3$ 次重复。10 月 1 日机械精量 播种, 10 月 7 日出苗。

\section{2 冠层光谱测定}

采用 MSI200 型成像光谱仪和 ASD Fieldspc FR2500 型光谱仪同步测定。MSI200 型成像光谱仪 由光学成像系统、可调谐液晶滤光器、高分辨低噪 声数字 CCD 像机和信息加工与处理系统组成, 通过 便携式计算机操作成像光谱仪进行数据采集与传输, 在 40 50 s 内即可完成 31 幅窄波段光谱图像的连续 采集; 数据传输方式为 USB 2.0 。成像光谱范围为 $420 \sim 720 \mathrm{~nm}$, 光谱分辨率为 $10 \mathrm{~nm}$, 感光器件为 $2 / 3$ 英寸 CCD, 分辨率为 $1280 \times 1024$, 即 131 万像素。 仪器主要特点是对目标物自 $420 \mathrm{~nm}$ 至 $720 \mathrm{~nm}$ 波段 范围, 每隔 $10 \mathrm{~nm}$ 分别成像并同时获取目标物在该 波段范围的光谱数据(即图像立方体)。对所获图像光 谱的定量分析, 可以直观分析成像范围内任意点的 光谱和颜色并进行目标物分类与识别。美国 ASD 公 司生产的 ASD Fieldspc Pro 系列光谱仪是国际公认 的性能稳定、用户最多的地物光谱仪，以其测定的 光谱反射率作为参照, 检验 MSI200 成像光谱仪测 定数据的可靠性。该仪器光谱范围为 $350 \sim 2500 \mathrm{~nm}$, 光谱分辨率在 350 1 $000 \mathrm{~nm}$ 区间为 $3 \mathrm{~nm}$, 在 $1000 \sim$ $2500 \mathrm{~nm}$ 区间为 $10 \mathrm{~nm}$ 。

选择晴朗无风日, 于 10:00-14:00 进行光谱数 据采集。MSI200 型成像光谱仪的视场角为 $14^{\circ}$, 镜 头放置于垂直距冠层顶部 $150 \mathrm{~cm}$ 处测定(图 1)。ASD FieldSpec FR2500 型光谱仪测量视场角为 $25^{\circ}$, 为与 成像光谱仪测定视场范围保持基本一致，探头置于 垂直距冠层顶部 $80 \mathrm{~cm}$ 处测定(图 2), 每个样点采集 20 条光谱数据, 计算平均值。用 MSI200 型光谱仪 测定时，在视场内放置白板同步测量其 DN 值作为 每幅图像光谱的参考。用 ASD 光谱仪测定光谱时, 每个点测定前后, 同步测定白板光谱反射率。目标 物的光谱反射率采用下式计算。

$$
R_{T}^{n}=\frac{D N_{T}^{n}}{D N_{R}^{n}} \times R_{R}^{n}
$$

式中, $R_{T}^{n}$ 和 $R_{R}^{n}$ 分别为目标和参考板在第 $n$ 个 波段的光谱反射率, $D N_{T}^{n}$ 和 $D N_{R}^{n}$ 为目标和参考板在 第 $n$ 个波段反射的太阳光强度。

\section{3 叶绿素的测定}

采用UV1800 分光光度计, 以丙酮法测定叶绿 素含量 ${ }^{[5]}$ 。

冠层叶绿素密度 (canopy chlorophyll density， $\mathrm{CCD})$ 的计算公式为 
$\mathrm{CCD}\left(\mathrm{mg} \mathrm{cm}^{-2}\right)=$ 叶绿素含量 $\left(\mathrm{mg} \mathrm{g}^{-1}\right) \times$ 单位面 积总绿叶鲜重 $\left(\mathrm{g} \mathrm{cm}^{-2}\right)$ 。

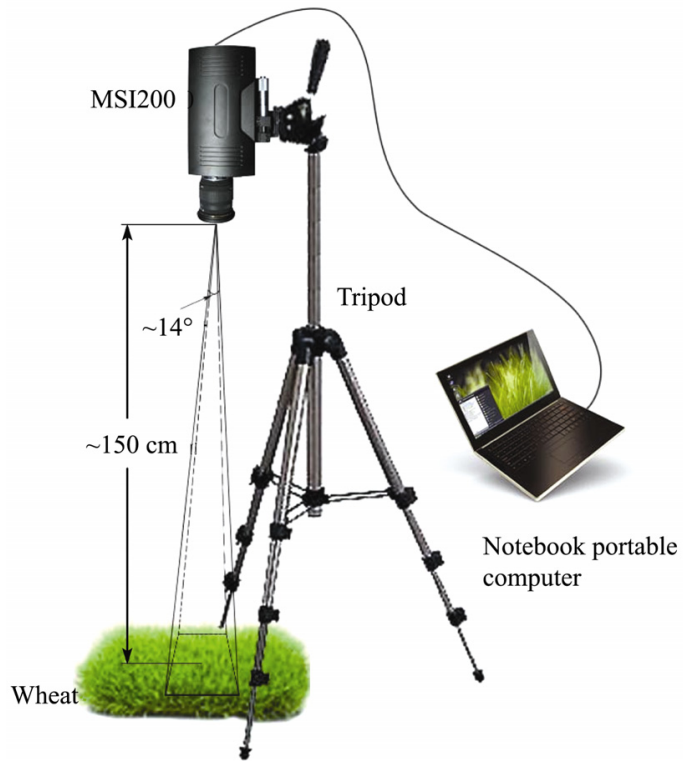

图 1 MSI200 成像光谱采集示意图

Fig.1 The sketch map of wheat canopy image and spectrum collection by MSI200

1.4 光谱特征参量的选择及数据分析

以包含绿色植被信息最多的可见光波段 (420 720 nm)作为筛选光谱特征的波段范围, 利用 作物光谱反射率及其数学变换和代数组合, 构建反 映作物叶绿素相关指标的光谱特征参量(表 1)。

采用 Matlab 7.0 和 SAS9.0 软件进行数据处理与 分析。

\section{2 结果与分析}

\subsection{MSI200 成像光谱仪数据特点}

MSI200 型成像光谱仪所获数据包括自 $420 \mathrm{~nm}$ 至 $720 \mathrm{~nm}$ 每 $10 \mathrm{~nm}$ 一幅图像及其相应的光谱值。分别

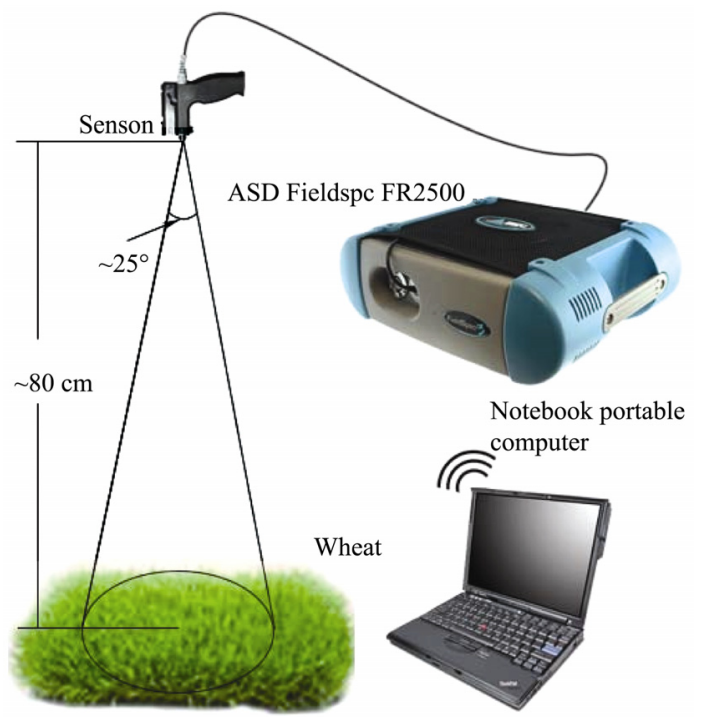

图 2 ASD Fieldspc FR2500 光谱采集示意图

Fig.2 The sketch map of wheat canopy spectrum collection by ASD Fieldspc FR2500

选择叶绿素高反射波段(550 nm)和强吸收波段(680 $\mathrm{nm}$ )以及全部波段合成图像(图 3 图 5)进行分析。2 种 仪器测定的目标物光谱, 视场内均包含小麦、土壤 等, 所得光谱数据为多种地物的混合光谱。相对于 ASD 光谱仪, MSI200 型成像光谱仪因能成像，可通 过图像明确地物目标，并得到该地物纯光谱信息， 利用该信息进行地物分类识别并成图(图 6)。结果表 明，利用这种方法进行小麦叶片识别与成图时，对 阴影和土壤等背景噪声有明显抑制效果。

\subsection{MSI200 成像光谱仪数据验证}

将 2 种仪器同一视场所得混合光谱数据进行 比较(图 7), 发现同一目标物的光谱反射率不同, 但波 形基本相同。MSI200 成像光谱仪测得的光谱反射率 高于 ASD Fieldspc FR2500 光谱仪。这一结果反映出 2 种仪器测定的目标物 DN 值相同, 只是在计算目标

表 1 本试验所选择的光谱特征参量

Table 1 Spectral parameters selected in the study

\begin{tabular}{lll}
\hline \multicolumn{1}{c}{$\begin{array}{c}\text { 光谱特征参量 } \\
\text { Spectral parameter }\end{array}$} & \multicolumn{1}{c}{$\begin{array}{c}\text { 计算公式 } \\
\text { Formula }\end{array}$} & $\begin{array}{c}\text { 参考文献 } \\
\text { Reference }\end{array}$ \\
\hline 单波段反射率 $\mathrm{R}$ & \multicolumn{1}{c}{$\mathrm{Ri}$} & Rouse et al. $(1974)^{[6]}$ \\
归一化植被指数 NDVI & $\mathrm{NDVI}=\left(\mathrm{R}_{720}-\mathrm{R}_{660}\right) /\left(\mathrm{R}_{720}+\mathrm{R}_{660}\right)$ & Gamom $(1992)^{[7]}$ \\
光化学反射指数 PRI & $\mathrm{PRI}=\left(\mathrm{R}_{570}-\mathrm{R}_{531}\right) /\left(\mathrm{R}_{570}+\mathrm{R}_{531}\right)$ & Metternicht $(2003)^{[8]}$ \\
比值植被色素指数 PPR & $\mathrm{PPR}=\left(\mathrm{R}_{550}-\mathrm{R}_{450}\right) /\left(\mathrm{R}_{550}+\mathrm{R}_{450}\right)$ & Gitelson et al. $(1996)^{[9]}$ \\
绿度归一化植被指数 GNDVI & $\mathrm{GNDVI}=\left(\mathrm{R}_{720}-\mathrm{R}_{550}\right) /\left(\mathrm{R}_{720}+\mathrm{R}_{550}\right)$ & Schleicher et al. $(2001)^{[10]}$ \\
氮素反射指数 NRI & $\mathrm{NRI}=\left(\mathrm{R}_{570}-\mathrm{R}_{670}\right) /\left(\mathrm{R}_{570}+\mathrm{R}_{670}\right)$ & Daughtry $(2000)^{[11]}$ \\
转换叶绿素吸收反射指数 TCARI & $\mathrm{TCARI}=3 \times\left[\left(\mathrm{R}_{700}-\mathrm{R}_{670}\right)-0.2 \times\left(\mathrm{R}_{700}-\mathrm{R}_{550}\right)\left(\mathrm{R}_{700} / \mathrm{R}_{670}\right)\right]$ & Penuelas et al. $(1995)^{[12]}$ \\
一阶微分 $\mathrm{R}^{\prime}(\Lambda \mathrm{i})$ & $\mathrm{R}{ }^{\prime}(\Lambda \mathrm{i})=\left[\left(\mathrm{R}_{\Lambda \mathrm{i}+1}-\mathrm{R}_{\Lambda \mathrm{i}-1}\right)\right] /\left[\left(\Lambda_{\mathrm{i}+1}-\Lambda_{\mathrm{i}-1}\right)\right]$ &
\end{tabular}




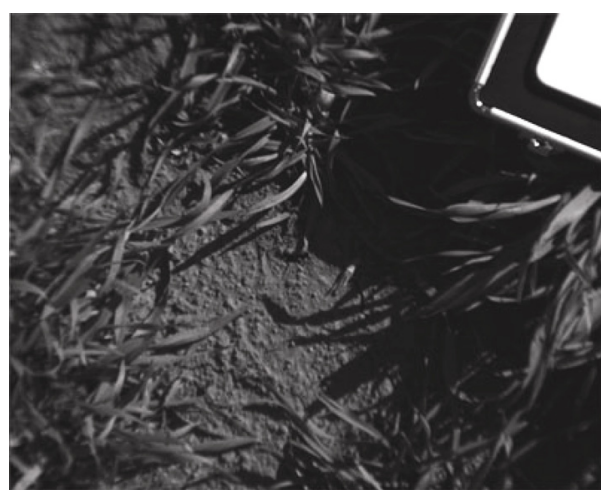

图 3 冬小麦 $550 \mathrm{~nm}$ 波段光谱图像

Fig. 3 Spectrogram of wheat at $550 \mathrm{~nm}$

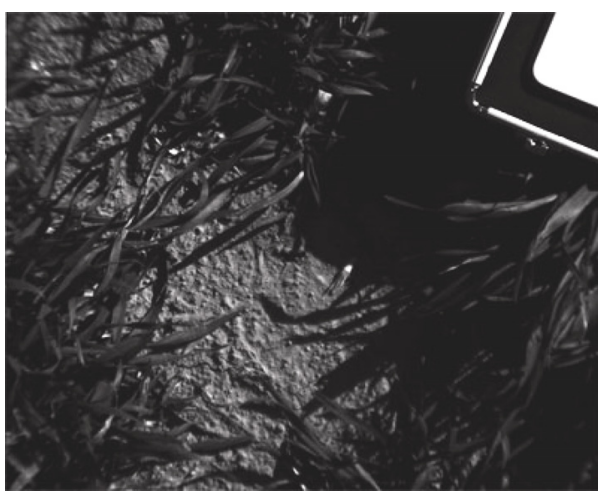

图 4 冬小麦 $680 \mathrm{~nm}$ 波段光谱图像

Fig. 4 Spectrogram of wheat at $680 \mathrm{~nm}$

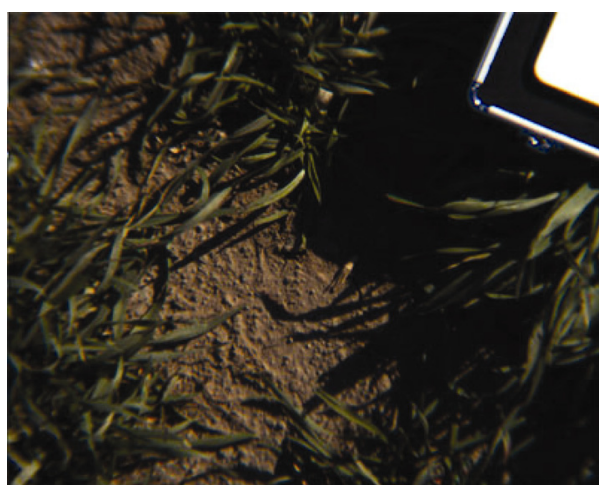

图 5 冬小麦各波段光谱合成图像

Fig. 5 Synthetic spectrogram of wheat from 420 to $720 \mathrm{~nm}$

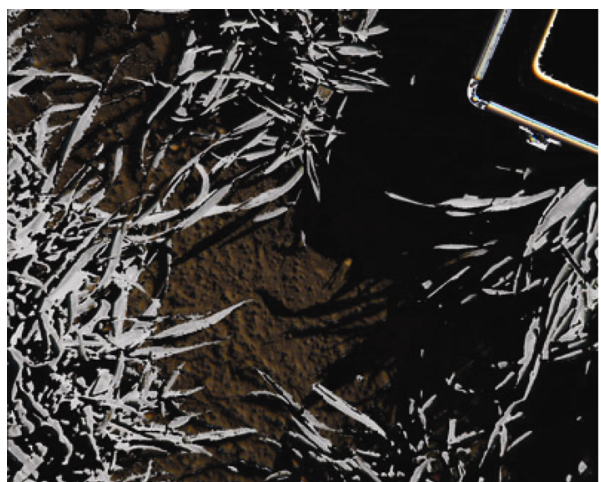

图 6 冬小麦冠层光谱分类图

Fig. 6 Purified spectrogram of wheat canopy from synthetic spectrogram

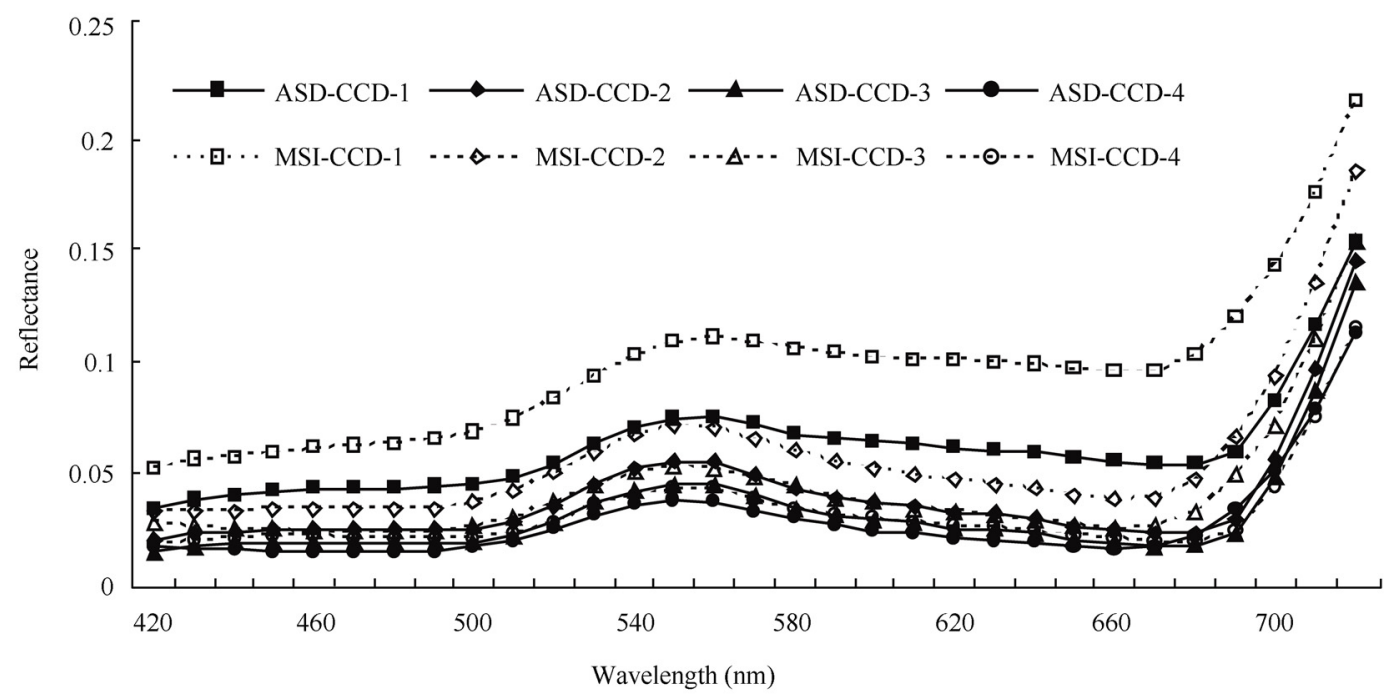

图 7 不同 CCD 梯度下 MSI200 与 ASD Fieldspc FR2500 反射光谱曲线

Fig. 7 Reflectance spectrum curve of wheat canopy at different CCD levels

物反射率时所用的参比有差异。这种差异可通过统计 方法和矫正模型加以消除, 并将 MSI200 型光谱仪反 射率数据矫正至与 ASD 反射率数据相同的水平。

为进一步检验 2 种仪器测定数据是否具有一致
性，对测定的多种处理的反射率数据进行拟合，两 仪器同一目标物的反射率值极显著相关 $(P<0.01)$ 。在 利用光谱数据进行作物生理生化参数反演时, 很少 直接用光谱反射率值, 更多的是通过反射率数学变 
换构建的光谱特征参量来估算。将光谱反射率变换 为表 1 所列光谱特征参量后, 2 种仪器获得的同一目 标物的同一光谱特征参量间的相关系数大于 0.9990 , 表明 MSI200 成像光谱仪所获光谱数据可靠。

\subsection{MSI 200 成像光谱仪监测叶绿素密度}

相关分析表明, 2 种仪器所获光谱参量均以 720 $\mathrm{nm}$ 的光谱反射率、 $550 \mathrm{~nm}$ 和 $660 \mathrm{~nm}$ 的一阶导数、 比值植被色素指数 $\operatorname{PPR}(550,450)$ 、绿度植被指数 GNDVI、氮素反射指数 NRI $(670,570)$ 以及转换叶绿 素吸收反射指数 TCARI 与 CCD 极显著相关(表 2), 可以较好地反映冠层叶绿素密度。

选择与 CCD 显著相关的光谱特征参量建立 $\mathrm{CCD}$ 预测模型，并进行精度检验。结果表明(表 3，表 4), 预测值与实测值间的相关系数达显著或极显著 水平。且基于 MSI 200 所建模型的均方根误差小于 0.2 ，相对误差小于 $9 \%$ ，而基于 ASD Fieldspc FR2500 所建模型的均方根误差介于 $0.8 \sim 1.3$ 之间,
表 2 小麦冠层叶绿素密度与光谱特征参量的相关系数

Table 2 Correlation coefficients between wheat canopy chlorophyll density and spectral parameters

\begin{tabular}{lcc}
\hline 光谱特征参数 & 相关系数 & Correlation coefficient \\
\cline { 2 - 3 } Spectral parameter & MSI 200 & $\begin{array}{c}\text { ASD Fieldspc } \\
\text { FR2500 }\end{array}$ \\
\hline R450 & -0.4685 & -0.4336 \\
R550 & -0.5001 & -0.5047 \\
R660 & -0.4891 & -0.4334 \\
R720 & $-0.8819^{* *}$ & $-0.8207^{* *}$ \\
R'450 & -0.3259 & -0.3041 \\
R'550 & $-0.5735^{*}$ & $-0.5293^{*}$ \\
R'660 & $0.8319^{* *}$ & $0.8069^{* *}$ \\
R'720 & -0.4548 & -0.2769 \\
PPR(550, 450) & $0.8916^{* *}$ & $0.8746^{* *}$ \\
TCARI & $-0.5533^{*}$ & $-0.5113^{*}$ \\
NDVI(720,660) & 0.3688 & 0.3442 \\
PRI(570, 530) & -0.2266 & -0.2070 \\
GNDVI & $0.8104^{* *}$ & $0.7894^{* *}$ \\
NRI(570, 670) & $-0.5923^{*}$ & $-0.5341^{*}$ \\
\hline${ }^{*}:$ significant at $P<0.05,{ }^{* *}:$ significant at $P<0.01$.
\end{tabular}

表 3 基于 MSI200 的小麦冠层叶绿素密度回归模型及检验

Table 3 Regression models and their tests for wheat canopy chlorophyll density data collected by MSI200

\begin{tabular}{|c|c|c|c|c|}
\hline $\begin{array}{l}\text { 光谱特征参数 } \\
\text { Spectral parameter }\end{array}$ & $\begin{array}{c}\text { 回归模型 } \\
\text { Regression model }\end{array}$ & $R^{2}$ & $\begin{array}{c}\text { 相对误差 } \\
\text { Relative error (\%) }\end{array}$ & $\begin{array}{c}\text { 均方根误差 } \\
\text { Root mean square error }\end{array}$ \\
\hline PPR & $y=0.4286 x-0.0818$ & $0.7949^{* *}$ & 4.46 & 0.0979 \\
\hline TCARI & $y=0.2718 \mathrm{e}^{-12.905 x}$ & $0.6632^{* *}$ & 8.53 & 0.1127 \\
\hline GNDVI & $y=0.0126 \mathrm{e}^{3.8725 x}$ & $0.7196^{* *}$ & 7.92 & 0.1266 \\
\hline NRI & $y=0.0395 \mathrm{e}^{-2.3763 x}$ & $0.6902^{* *}$ & 8.10 & 0.1318 \\
\hline $\mathrm{R} 720$ & $y=-0.5498 x+0.164$ & $0.6941^{* *}$ & 8.62 & 0.1409 \\
\hline $\mathrm{R}^{\prime} 550$ & $y=-129.89 x+0.0941$ & $0.5984^{* *}$ & 8.74 & 0.1570 \\
\hline$R^{\prime} 660$ & $y=1649.8 x+0.1273$ & $0.6920^{* *}$ & 8.32 & 0.1199 \\
\hline
\end{tabular}

${ }^{*}$ : significant at $P<0.05,{ }^{* *}:$ significant at $P<0.01$. Samples for model-establishment and test were 25 and 20 , respectively.

表 4 基于 ASD FR2500 的小麦冠层叶绿素密度回归模型及检验

Table 4 Regression models and their tests for wheat canopy chlorophyll density data collected by ASD Fieldspc FR2500

\begin{tabular}{|c|c|c|c|c|}
\hline $\begin{array}{c}\text { 光谱特征参数 } \\
\text { Spectral parameter }\end{array}$ & $\begin{array}{c}\text { 回归模型 } \\
\text { Regression model }\end{array}$ & $R^{2}$ & $\begin{array}{c}\text { 相对误差 } \\
R E(\%)\end{array}$ & $\begin{array}{c}\text { 均方根误差 } \\
\text { RMSE }\end{array}$ \\
\hline PPR & $y=0.3414 x-0.0471$ & $0.6737^{* *}$ & 7.21 & 0.8391 \\
\hline TCARI & $y=2.1260 x-0.1087$ & $0.6114^{* *}$ & 11.40 & 0.9795 \\
\hline GNDVI & $y=0.0170 \mathrm{e}^{3.2166 x}$ & $0.6243^{* *}$ & 10.25 & 0.9982 \\
\hline NRI & $y=0.0343 \mathrm{e}^{-2.2775 x}$ & $0.5539^{* *}$ & 11.17 & 1.2533 \\
\hline $\mathrm{R} 720$ & $y=0.3186 x+0.0284$ & $0.5447^{* *}$ & 12.48 & 1.0175 \\
\hline $\mathrm{R}^{\prime} 550$ & $y=-156.02 x+0.0976$ & $0.4656^{*}$ & 13.61 & 1.0002 \\
\hline $\mathrm{R}^{\prime} 660$ & $y=-994.74 x-0.0854$ & $0.6232^{* *}$ & 13.39 & 0.9069 \\
\hline
\end{tabular}

": significant at $P<0.05,{ }^{* *}:$ significant at $P<0.01$. Samples for model-establishment and test were 25 and 20 , respectively. 
相对误差介于 $7 \%$ 14\%之间。两仪器所得模型中均 以比值植被色素指数 PPR 的模型预测误差最小，精 度最高; 并且基于 MSI 200 的模型精度明显优于 ASD Fieldspc FR2500, 能够更好地对小麦苗期叶绿素密 度进行估测。

\section{3 讨论}

目前在近地高光谱遥感中所采用的仪器几乎全 部是非成像地物光谱仪，其基本工作原理是通过光 导探头摄取目标光线及目标物的光辐射值, 经过模/ 数转换器变成数字信号, 得到目标物的光谱信息。 在数据采集过程中获得的是探头视场范围内所有物 体的混合光谱，在进行数据应用时很难剔除背景等 干扰物的光谱信息，降低了监测精度，并且容易出 现“同物异谱”和“同谱异物”现象。成像光谱仪不仅 能够获取目标物的光谱信息，同时还可获得其图像 数据。图像是传递信息的最佳方式，它不仅直接给 出目标形状和位置, 还可以了解目标物同背景及其 他物体的相互关系。使用成像光谱仪能够获取比较 精细的纯光谱信息，提高遥感数据的定量水平。

本文利用成像光谱数据, 通过去混合、光谱分 类等方法，提取了小麦冠层中的植株光谱反射率， 用相应光谱特征参量对小麦冠层叶绿素密度进行了 遥感监测, 所建立的回归模型均达显著或者极显著 水平, 预测精度较高、误差较小。并且由于成像光 谱仪获取的是面状数据, 与传统非成像地物光谱仪 获得的点状数据相比, 不仅能够通过构建和篮选重 要植被指数或光谱指数实现简化波段, 而且对于航 天、航空遥感器定标和“星一机一地”一体化同步观 测有重要意义。在本试验过程中, 发现成像光谱仪 在测定时依然受到天气、光强、风速和定标物等外 界因素的影响, 如何选择最佳的测试条件显得尤为 重要, 对此仍需要进一步探索和研究。

\section{4 结论}

以 ASD Fieldspc FR2500 光谱仪为对照, MSI200 成像光谱仪所获得的小麦苗期冠层光谱 DN 值与之 相同, 两仪器光谱曲线波形一致, 反射率值及相应 光谱特征参量达极显著相关水平, MSI200 成像光谱 数据真实、可靠，能够用于地物波谱信息的测量。 MSI200 成像光谱仪能够更精确地对冬小麦苗期冠 层叶绿素密度进行监测, 所得模型的 RMSE 为 $0.0979, \mathrm{RE}$ 为 $4.46 \%$, 比值植被色素指数 PPR 可以作
为最佳监测指标。

\section{References}

[1] Ji H-Y(吉海彦), Wang P-X(王鹏新), Yan T-L(严泰来). Estimations of chlorophyll and water contents in live leaf of winter wheat with reflectance spectroscopy. Spectroscopy \& Spectral Anal (光谱学与光谱分析), 2007, 27(3)：514-516 (in Chinese with English abstract)

[2] Zhao X(赵祥), Liu S-H(刘素红), Wang P-J(王培娟), Wang J-D(王锦地), Tian Z-K(田振坤). A method for inverting chlorophyll content of wheat using hyperspectra. Geography \& Geo-Inf Sci (地理与地理信息科学), 2004, 20(3): 36-39 (in Chinese with English abstract)

[3] Pu R-L(浦瑞良), Gong P(宫鹏). Hperspectral Remote Sensing and Its Applications (高光谱遥感及其应用). Beijing: Higher Education Press, 2000. pp 25-48(in Chinese)

[4] Curran P J, Dungan J L, Macler B A, Plummer S E, Peterson D L. Reflectance spectroscopy of fresh whole leaves for the estimation of chemical concentration. Remote Sens Environ, 1992, 39: $153-166$

[5] Zhang X-Z(张宪政). A comparison of methods of measurement of chlorophyll in plants. J Shenyang Agric Coll (沈阳农业大学 学报), 1985, 16(4): 81-84 (in Chinese with English abstract)

[6] Rouse J W, Haas R H, Schell J A, Deering D W, Harlan J C. Monitoring the Vernal Advancement of Retrogradation of Natural Vegetation, NASA/GSFC, Type III, Final Report, Greenbelt, MD, USA. 1974

[7] Gamon J A, Penuelas J, Field C B. A narrow-waveband spectral index that tracks diurnal changes in photosynthetic efficiency. Remote Sens Environ, 1992, 41: 35-44

[8] Metternicht G. Vegetation indices derived from highresolution airborne videography for precision crop management. Int $J$ Remote Sens, 2003, 24: 2855-2877

[9] Gitelson A A, Kaufman Y J, Merzlyak M N. Use of a green channel in remote sensing of global vegetation from EOSMODIS. Remote Sens Environ, 1996, 58: 289-298

[10] Schleicher T D, Bausch W C, Delgado J A, Ayers P D. Evaluation and refinement of the nitrogen reflectance index (NRI) for site-specific fertilizer management. In: ASAE Annual International Meeting Report, ASAE Paper No. 01-11151. St. Joseph, MI, USA, 2001

[11] Daughtry C S T, Walthall C L, Kim M S, Brown de Colstoun E, McMurtrey J E. Estimating corn foliar chlorophyll content from leaf and canopy reflectance. Remote Sens Environ, 2000, 74: 229-239

[12] Peñuelas J, Filella I, Gamon J A. Assessment of photosynthetic radiation-use efficiency with spectral reflectance. New Phytol, 1995, 131: 291-296 